\title{
Development of OFDM based Secondary Link: Some Experimental Results on USRP N210 Platform
}

\author{
Miloš Janjić, Milenko Brković, and Miljko Erić
}

\begin{abstract}
Some experimental results of the concept development and practical implementation of an Orthogonal Frequency Division Multiplexing (OFDM) based secondary cognitive link are presented in this paper. The secondary link is realized using Universal Software Radio Peripheral (USRP) N210 platforms. For communication with USRP, we use MATLAB toolbox. Several algorithms are used to overcome transmission problems. Time-synchronization is achieved using a method based on auto-correlation of two sliding windows. Frequency offset estimation is performed using a phase offset between samples in a signal header, comprised of a sinusoid. A channel is estimated using predefined symbols inserted at the beginning of every frame, which enables channel equalization. Also, the cognitive feature of spectrum sensing and changing transmission parameters is implemented. A least-mean-square adaptive filter is introduced to offer time-synchronization error estimation as well as an alternative option for channel equalization.
\end{abstract}

Keywords - Cognitive radio, OFDM, secondary link, spectrum sensing, USRP platform.

\section{INTRODUCTION}

$\mathrm{T}$ HE growth of wireless communication has created new technologies, which provide services with a high bandwidth and lead to an increase in the usage of radio spectrum. It was reported that some parts of the allocated spectrum at some locations were unused most of the time [1]. The scarcity of spectrum and need for efficient usage have prompted research aimed at finding some other, more flexible, way of spectrum use, which has led to the development of a cognitive radio concept [2]. The basic idea is to make the spectrum access more flexible, i.e., to allow unlicensed users to use the licensed spectrum, with

Paper received March 24, 2014; accepted June 14, 2014. Date of publication July 31,2014 . The associate editor coordinating the review of this manuscript and approving it for publication was Prof. Aleksandar Nešković.

This paper is a revised and expanded version of the paper presented at the 21th Telecommunications Forum TELFOR 2013.

This work was supported by Serbian Ministry of Science, Education and Technological Development under project TR32028 - "Advanced Techniques for Efficient Use of Spectrum in Wireless Systems"

Miloš Janjić, Student of PhD study, University of Belgrade - School of Electrical Engineering, milos_janjic@yahoo.com.

Milenko Brković, Student of PhD study, University of Belgrade School of Electrical Engineering, Telenor, milenkobrkovic@gmail.com.

Miljko Erić, University of Belgrade - School of Electrical Engineering, meric@open.telekom.rs. an obligation to obey some rules. In the cognitive radio (CR) terminology, licensed users are called primary users, while unlicensed users are called secondary or cognitive users. The main aim of $\mathrm{CR}$ is to enable dynamic spectrum access, thereby the adjustment of radio systems to environment in the way that optimizes overall system performances, while improving the utilization of the spectrum [3].

Therefore, a secondary link must be able to dynamically change transmission parameters in order not to make interference towards primary users. The practical implementation of CR has been achieved by Software Defined Radio (SDR) [4]. SDR technology provides implementation of a radio communication system in which the components implemented on hardware are transferred to software that provides the flexibility of changing the operating parameters of the device like bandwidth, central frequency, modulation scheme, etc. In fact, CR is defined as "intelligence" that sits above SDR and lets SDR determine which mode of operation and parameters to use [5].

The most important task in CR is spectrum sensing, that includes the process of spectrum holes detection and the analysis of signals active in some part of the spectrum (modulation type, bandwidth, carrier frequency, etc.). While searching for spectrum holes, a few dimensions of the spectral space are investigated, such as time, frequency, location, angle of arrival, code, etc.

Orthogonal Frequency Division Multiplexing (OFDM) modulation technique is a good choice for the implementation of a cognitive secondary link primarily because of its scalability. It is possible to dynamically change parts of spectrum where the presence of primary users is detected simply by introducing zero subcarriers at the corresponding positions. In addition, the processes of modulation and demodulation use the IFFT and FFT algorithms whose execution is optimized by today's fieldprogrammable gate array (FPGA) chips.

Some first experimental results of the development and practical implementation of an OFDM based secondary cognitive link using Universal Software Radio Peripheral (USRP) N210 platforms are presented in this paper.

The second section presents our experimental infrastructure. The third section describes the method of implementation of the secondary link and explains all the algorithms that were used. Section four contains the obtained experimental results. The future steps in the 
development of the secondary link are at the end of the paper.

\section{DeVELoPMent ENVIRONMENT}

This section discusses the hardware and software platform used for our solution. USRP (Ettus Research, USA) was used as SDR. USRP provides a digital baseband and IF section within the hardware, which aids utilizing general purpose computers to function as high bandwidth software radios. In addition, the board can provide interface to various daughter-boards for a wide range of applications. The basic philosophy behind the USRP is to provide all waveform specific features like modulation and demodulation in CPU, whereas the high speed operations like interpolation, decimation, digital up and down conversion are provided within FPGA [6].

For communication with USRP, the MATLAB toolbox for USRP was used. The MATLAB toolbox communicates with the USRP platform using the UDP (User Datagram Protocol) protocol. A computer sends OFDM baseband signal samples to the USRP which generates a signal at a given frequency and bandwidth specified by an interpolation factor. The signal is then transmitted over an antenna to the receiving end where all operations are reversely executed.

\section{IMPLEMENTATION}

In the implementation we have started from an OFDM simulator published in [7]. It is a baseband OFDM simulator, which therefore employs only a half of subcarriers for useful data transmission, while the other half is conjugate subcarriers. We implemented the following features: pass-band communication employing all subcarriers for useful data transmission, improved time synchronization, frequency offset estimation and compensation, demodulation of frame data immediately upon its arrival at the transmitter and display of corresponding image data, cognitive property by performing spectrum sensing and changing carrier frequency, channel estimation and equalization, QAM modulation, and estimation of time-synchronization errors using an adaptive filter.

Source data is taken from an 8-bit grayscale (256 gray levels) bitmap image file, shown in Fig. 2, as in [7]. The image data is then converted to the symbol size determined by the choice of $M$-PSK. The converted data is then separated into multiple frames by the OFDM transmitter. The OFDM modulator modulates the data frame by frame. During modulation symbols are differentially encoded. Before the exit of the transmitter, the modulated frames of time signal are cascaded together along with frame guards inserted in between as well as a pair of identical headers consisting of a sinusoid added to the beginning and end of the data stream. A cyclic prefix length is $25 \%$ of the useful OFDM symbol length, the length of guard intervals inserted equals the length of an OFDM symbol with a cyclic prefix, while header and trailer size is 8 OFDM symbols.

\section{A. M-DPSK coding}

M-DPSK modulation is deployed on every subcarrier, as in [7]. While transmitting our 256-grayscale bitmap image, there is one benefit of using 256-DPSK modulation. Namely, if adjacent levels of grey are coded with adjacent constellation points, then small mistakes in demodulation (identifying some adjacent constellation point instead of the right one) result in small mistakes in grayscale levels interpretation, which is not easily observed by human eye. Errors are easily noticed only in the case of white and black pixels, because they are adjacent in the constellation.

\section{B. Time synchronization}

Time synchronization processing recognizes the presence of the signal on the input of the receiver. We use a method based on auto-correlation of two sliding windows of length $N$, shifted in time [8]

$$
\begin{aligned}
a_{n} & =\sum_{k=0}^{N-1} r_{n-k} r_{n-k}^{*}=\sum_{k=0}^{N-1}\left|r_{n-k}\right|^{2} \\
b_{n} & =\sum_{k=0}^{N-1} r_{n+k} r_{n+k}^{*}=\sum_{k=0}^{N-1}\left|r_{n+k}\right|^{2} .
\end{aligned}
$$

Variable $m_{n}=a_{n} / b_{n}$ peaks when auto correlation $a_{n}$ measures the signal with noise, and $b_{n}$ only noise, where variable $r_{n}$ denotes the $n$-th sample of the received signal. The algorithm is illustrated in Fig. 1.

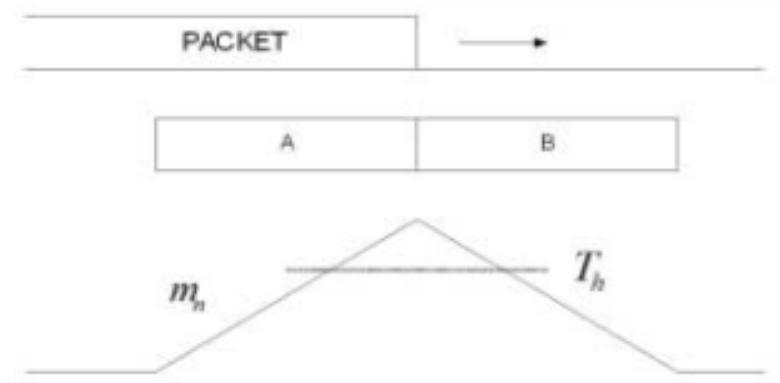

Fig. 1. Packet detection with sliding auto-correlations [8].

In the peak point, $m_{n}$ has a value $\max \left(m_{n}\right)=\frac{S+N}{N}=\frac{S}{N}+1$, so the detecting threshold could be set according to the expected SNR. First, rough synchronization is performed, where sliding windows are shifted by a predefined number of samples in every step. After that, fine synchronization takes place, where windows are shifted sample by sample as in [9]. After detecting the start of the header, the beginning of all the frames is calculated from the known structure of the signal, so the accuracy of detecting the start of the header is crucial.

\section{Frequency offset estimation and compensation}

Even if we intend to generate exactly the same carrier frequencies in the transmitter and receiver, there is a difference between them due to the physically inherent nature of the oscillators, called carrier frequency offset (CFO). As it is well known, OFDM systems are very sensitive to this phenomenon. The normalized CFO, $\varepsilon$, is 
defined as

$$
\varepsilon=\frac{f_{\text {offset }}}{\Delta f}
$$

where $f_{\text {offset }}$ denotes CFO, while $\Delta f$ denotes subcarrier spacing.

For the transmitted time domain signal $x[n]$, the CFO causes a phase offset of $2 \pi n \varepsilon / N$ in the received signal $y[n]$, i.e., $y[n]=e^{j 2 \pi n \varepsilon / N} x[n]$, where $N$ is FFT size, which is equivalent to a frequency shift of $\varepsilon$ in the frequency domain signal $X[k]$, i.e., $Y[k]=X[k-\varepsilon]$. Two time domain techniques, using a cyclic prefix (estimation range $[-0.5,0.5)$ ) and training symbols (a wider range), respectively, as well as two frequency domain techniques, using preamble, and pilot tones, respectively, are described in [10]. In [8] a 2-step time domain estimation is performed, using short symbols in preamble for rough estimation, and long symbols for fine offset recovery. In this paper, a header, comprised of a sinusoid, is used as a training sequence. Let $D$ denote a ratio between FFT size, $N$, and the sinusoid period. The estimated normalized CFO can then be expressed as

$$
\hat{\varepsilon}=\frac{D}{2 \pi} \arg \left\{\sum_{n=1}^{M} y^{*}[n] y[n+N / D]\right\}
$$

where $M$ denotes an arbitrary number of samples used in the calculation. After performing the estimation, samples of the time domain signal are multiplied by $e^{-j 2 \pi n \varepsilon / N}$, to compensate for the CFO. This realization is sensitive to noise. Note that dependency on the quality of time synchronization is avoided.

\section{Channel estimation and equalization}

Several predefined symbols are inserted at the beginning of every frame in order to estimate channel frequency response. The structure of these symbols is the same as of useful symbols. The impact of the channel on a transmitted signal is very complex, including noise, fading, attenuation, and after all it is time-varying. In this first iteration we use the simplest model of the channel represented with the frequency response of the channel. A frequency domain signal at the receiving end, $y(\omega)$ can be represented as

$$
y(\omega)=H(\omega) x(\omega)
$$

where $x(\omega)$ denotes the transmitted signal. Parameter $H(\omega)$ is the frequency response of the channel. At the beginning of every frame, for every predefined symbol the frequency response of the channel is calculated. Then the frequency response of the channel averaged over all predefined symbols is used to calculate average equalization function

$$
H_{e q}(\omega)=\frac{1}{H_{a v g}(\omega)} .
$$

By multiplying it with every useful OFDM symbol in the frequency domain, channel equalization is performed.

\section{E. QAM modulation}

At the transmitter, data bits are mapped to QAM symbols using simple binary coding. Because of still image transmission, Gray coding wouldn't bring any benefits. The phases of QAM symbols are differentially coded. At the receiver, channel estimation and equalization allow to set properly levels for the detection and demodulation of QAM symbols.

\section{EXPERIMENTAL RESULTS}

The following results were obtained by experiments conducted in a laboratory environment, deploying 2 laptops controlling the transmit and receive N210 USRP platforms, respectively, as shown in Fig. 3. Transmission parameters were: $400 \mathrm{MHz}$ carrier frequency, bandwidth of $1 \mathrm{MHz}$, OFDM with 1024 subcarriers.

Figs. 4 and 5 show images obtained in the receiver after transmission using 256 DPSK and 256 QAM modulations, respectively. The percent of incorrectly detected pixels in the case of 256 DPSK was 90.5, whereas in the case of 256 QAM it was 17.6. It is evident that subjectively 256 DPSK image seems to be almost perfect, although $90.5 \%$ of symbols were incorrectly detected, because of the earlier described benefit of mapping image bits to 256 PSK symbols.

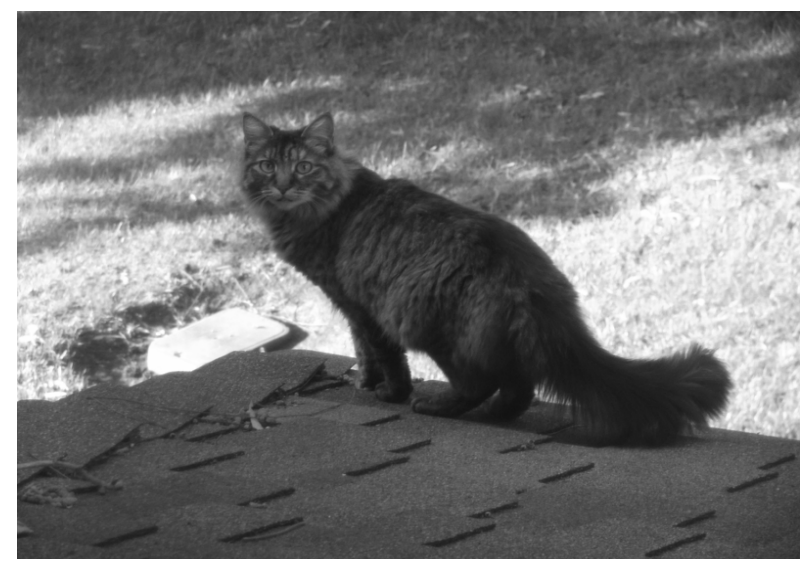

Fig. 2. 256-grayscale bitmap image transmitted in the experiments.

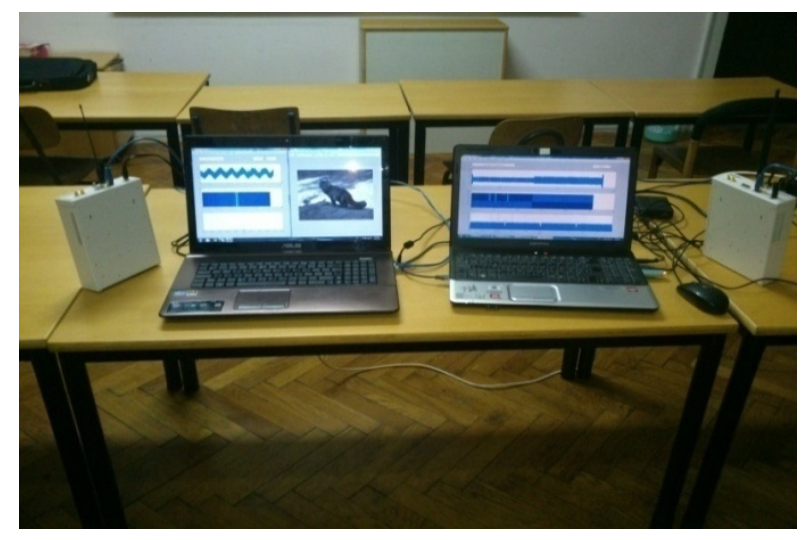

Fig. 3. Development environment.

With the aim of demonstrating the secondary link principle, in addition to the data signal (a secondary signal), another signal was introduced at the Tx. It was 
generated in the form of MATLAB file, and it emulated a primary user signal as seen from the Tx. This signal, comprised of 3 sinusoids and located in the lower half of the secondary signal frequency band, appeared at some random point in time, unknown to the $\mathrm{Tx}$, during the transmission of secondary user data. After the start, all parameters of this signal didn't change with time. In order to determine the moment of the appearance of primary user signal, spectrum sensing using an energy detector was performed at the Tx during guard time intervals.

The time domain secondary signal was interpolated by a factor of 2 in MATLAB, and having in mind that the sampling frequency of transmitted signal was unchanged, bandwidth of signal narrowed 2 times. The secondary user signal for transmission was created frame by frame. Starting with the first frame, signal spectrum was shifted to the lower subband.

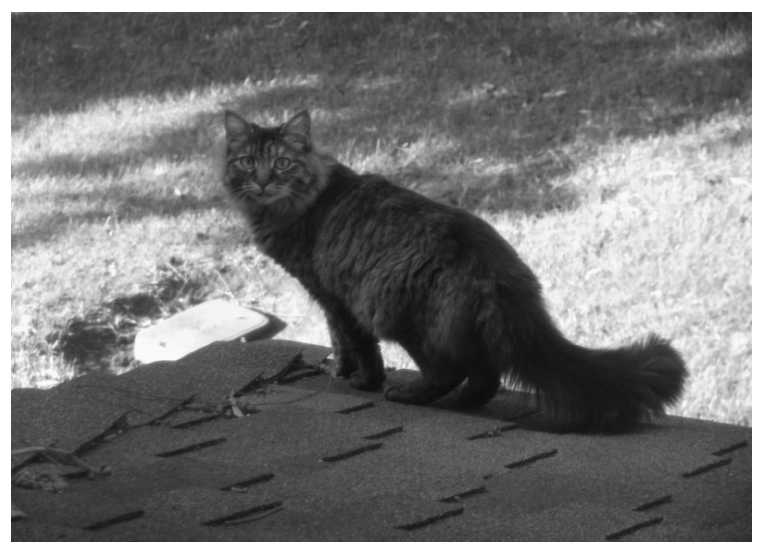

Fig. 4. Image in the receiver after transmission using 256 DPSK.

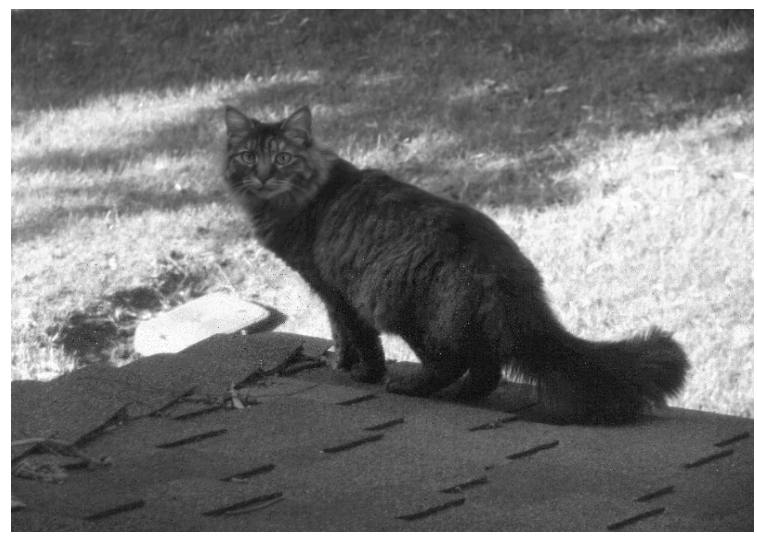

Fig. 5. Image in the receiver after transmission using 256 QAM.

After every frame of data, energy in a guard interval was measured and compared to a predefined threshold, which was close to zero. While a primary user hadn't been detected, transmission took place in the lower subband. When the primary user signal was detected, signal spectrum was shifted to the upper subband, in order to avoid interfering with a primary user. Because the parameters of the primary user signal didn't change, the rest of the data stream was transmitted in the upper subband. The transmission of the frame preceding the moment of detecting the primary user was repeated after changing subband.

Along with the secondary user data, primary user data were transmitted, as well, in order to emulate a primary user signal as seen from the $\mathrm{Rx}$, which enabled $\mathrm{Rx}$ to perform real spectrum sensing. This corresponds to a situation where secondary $\mathrm{Tx}$ and Rx "see" the same environment.

The Rx was informed about the subband where the signal transmission started, as that after primary user appearance change of secondary signal transmission subband took place, as well as, repeated transmission of the corrupted frame. The biggest challenge was to discover the moment of primary user appearance. For this purpose Rx performed spectrum sensing.

The receiver bandwidth equaled the original signal bandwidth. At the Rx side the structure of the transmitted signal was known. After every received frame, energy in a guard interval was measured and compared to a predefined threshold, set by measuring the noise level during the first guard interval placed between the header and the first frame of the data. If the threshold wasn't reached, the $\mathrm{Rx}$ considered that the previous frame of secondary signal had been emitted in the lower subband. When the threshold was reached, Rx "concluded" that a primary user had appeared during the reception of the previous frame. After that, the Rx considered that the secondary user signal was emitted in the upper subband.

The signal spectrum of every received frame of data was shifted back to the baseband, and unnecessary frequencies were filtered out, i.e., digital down conversion was performed. Then, decimation took place, signal spectrum was spread by a factor of 2 . As demodulation was performed frame by frame, and the image and signal spectrum displays were provided synchronously, the moment of the primary user appearance could be clearly noticed, as well as signal spectrum shifting and repeated display of the pixels belonging to the frame of data transmitted at the moment of primary user appearance. In this way the system was given the basic cognitive property. The described scenario is illustrated in Fig. 6.

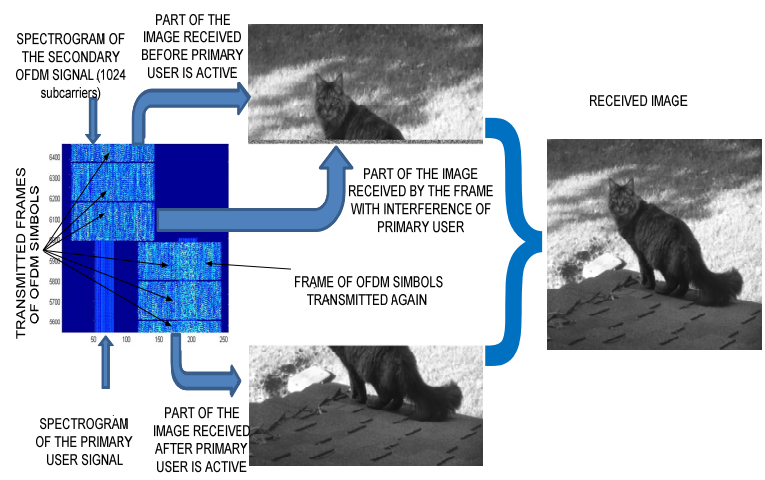

Fig. 6. Cognitive property implemented in secondary link.

Fig. 7 shows the real part of time domain baseband samples, the spectrogram and frequency spectrum of the RF signal at the transmitter, respectively. Fig. 8 gives the corresponding displays for the signal at the receiver. At the edges of the signal bandwidth, a slight decrease in 
spectrum level can be noticed, which is due to the characteristic of filters in the receive chain of USRP.
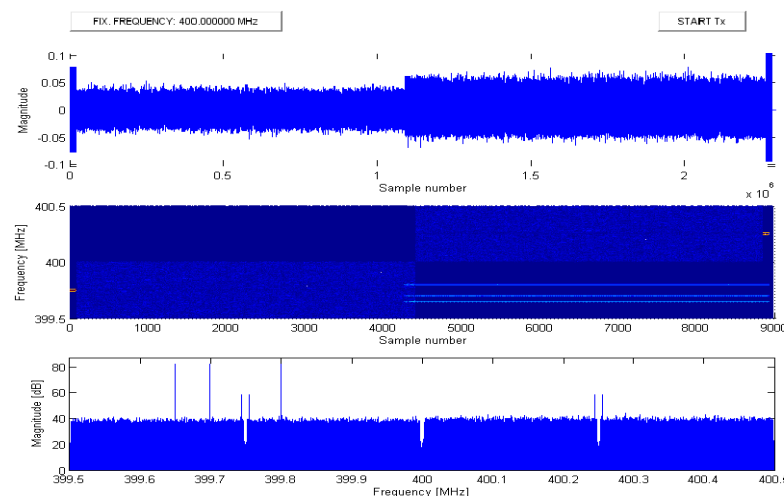

Fig. 7. Time domain samples, spectrogram, and spectrum of the transmitted signal.
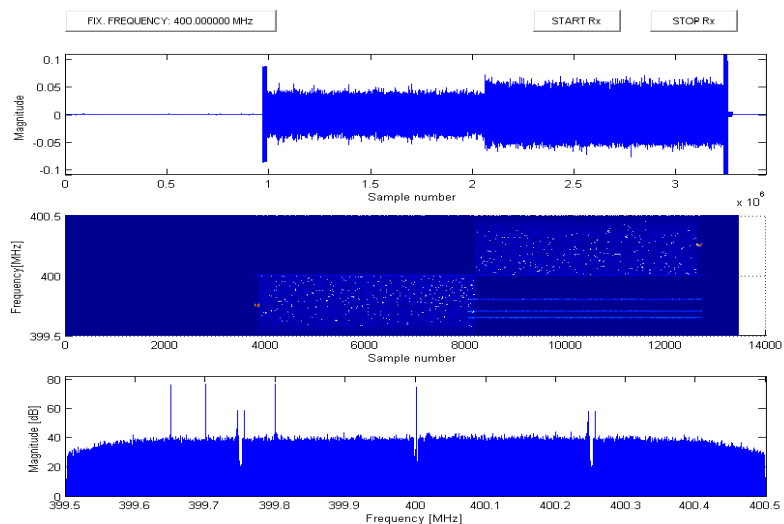

Fig. 8. Time domain samples, spectrogram, and spectrum of the received signal.

Fig. 9 gives the normalized estimated channel transfer function of the upper subband (decrease of spectrum level at the right edge) at one of the OFDM frames.

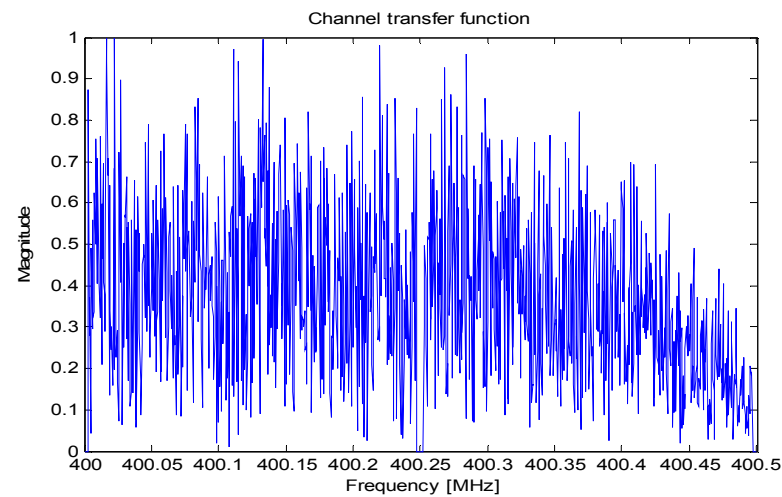

Fig. 9. Normalized channel transfer function at one of the frames.

Constellation diagrams for 256 DPSK modulation at the transmitter and receiver are given in Figs. 10 a) and b), respectively.

Constellation diagrams for 256 QAM modulation at the transmitter and receiver are given in Figs. 11 a) and b), respectively.

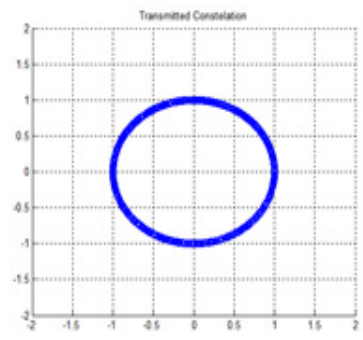

a)

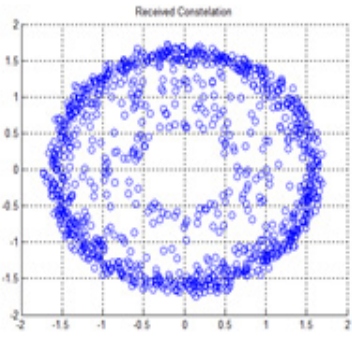

b)
Fig. 10. Constellation diagram for 256 DPSK modulation a) at the transmitter b) at the receiver after channel equalization.

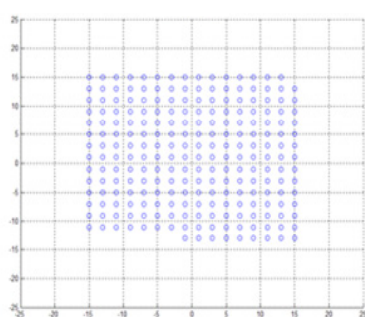

a)

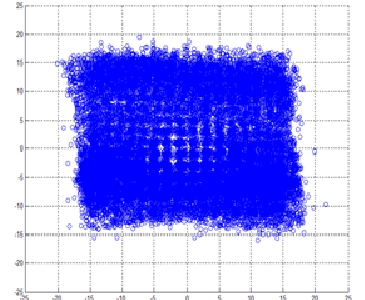

b)
Fig. 11. Constellation diagram for 256 QAM modulation a) at the transmitter b) at the receiver after channel equalization.

We used an adaptive filter with the Least Mean Square (LMS) algorithm [11] to estimate time synchronization errors. Previously described predefined symbols were used. At the $\mathrm{Rx}$, the part of time-domain signal corresponding to IFFT of these symbols was passed through an adaptive filter and compared to its original version created at the Tx. Synchronization error was determined from the impulse response of the adaptive filter. The adaptive filter can be used instead of the above described method for channel equalization, too. In this case, FFT of the adaptive filter impulse response (transfer function) is used as the equalization function. Fig. 12 shows the estimated channel transfer function of the lower subband (decrease of spectrum level at the left edge) at one of the OFDM frames, transfer function of the adaptive filter, as well as, overall transfer function, obtained by multiplying these two transfer functions.

\section{CONCLUSIONS AND FUTURE WORK}

In this paper our technical solution for a secondary link has been presented. We have managed to transmit picture in real conditions, overcoming a lot of problems that don't exist in software simulations of OFDM link, and also implementing a cognitive feature of spectrum sensing and changing transmission parameters. But there are still a large number of problems to be solved, which will be our future work. The next step in our project is a gradual increase in the speed of operation of the transmitter and receiver. Primarily, solving a problem of buffering samples from the USRP platform, a greater level of controllability of our application, because now communication between the USRP platform and computer is performed over the MATLAB toolbox, which is very 
slow (using UDP), and data is processed in MATLAB. Lowering at the MAC layer, the implementation of the whole mechanism in FPGA technology using the ML605 platform and NUTAC modules serving as an analog frontend, would be final steps to maximize the speed of the application and thus enable its use in real-life conditions.

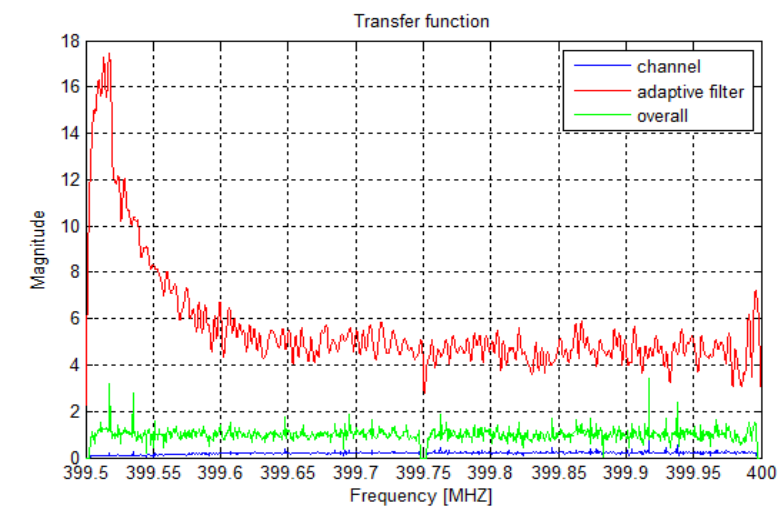

Fig. 12. Channel transfer function, adaptive filter transfer function, and overall transfer function.

\section{ACKNOWLEDGMENT}

The authors would like to thank Dr Jelena Ćertić for her contribution and useful suggestions regarding implementation of adaptive channel equalization.

\section{REFERENCES}

[1] F. F. Digham, "Joint power and channel allocation for cognitive radios," in Proc. Wireless Comm. Networking Conference, pp. 882887, Las Vegas, NV, USA, Apr., 2008.

[2] J. Mitola, "Cognitive radio: an integrated agent architecture for software defined radio," PhD thesis, Royal Institute of Technology (KTH), Stockholm, Sweden, 2000.

[3] D. B. Maldonado, B. Le, A. Hugine, T. W. Rondeau and C. W. Bostian, "Cognitive radio applications to dynamic spectrum allocation: a discussion and an illustrative example," in Proc. First IEEE Int. Symp. on New Frontiers in Dyn. Spectrum Access Networks, pp: 597-600, Baltimore, MD, USA, Nov. 2005.

[4] J. Mitola, G.Q. Maguire, "Cognitive radio: making software radios more personal," IEEE Pers. Comm., vol. 6, no. 4, pp. 13-18, Aug. 1999.

[5] R. W. Thomas, L. A. DaSilva, and A. B. MacKenzie "Cognitive networks," in Proc. First IEEE Int. Symp. on New Frontiers in Dyn. Spectrum Access Networks, Baltimore, MD, USA, Nov. 2005.

[6] V. V. Patcha, "Experimental study of cognitive radio test-bed using USRP", thesis by B. TECH in Electronics and Communication Engineering Padmasri Institute of Technology, India, Aug. 2011.

[7] P. G. Lin, "OFDM simulation in MATLAB", California Politechnic State University, San Luis Obispo, June 2010.

[8] D. M. Dramicanin, D. Rakic, S. Denic and V. Vlahovic, "FPGAbased prototyping of IEEE 802.11 a baseband processor", Serbian J. Elect. Eng., vol.1, no. 3, pp. 125-136, Nov. 2004.

[9] R. Van Nee, R. Prasad, OFDM for Wireless Multimedia Networks, Artech House, 2001.

[10] Y. S. Cho, J. Kim, W. Y. Yang, C. G. Kang, MIMO-OFDM Wireless Communications with MATLAB, Wiley, Singapore, 2010.

[11] J. Proakis, Digital Communications, 4th edition, McGraw Hill, 2001. 